LIVER

\title{
Quantitative gene expression in Budd-Chiari syndrome: a molecular approach to the pathogenesis of the disease
}

\author{
V Paradis, I Bièche, D Dargère, D Cazals-Hatem, I Laurendeau, V Saada, J Belghiti, \\ A Bezeaud, M Vidaud, P Bedossa, D-C Valla
}

See end of article for authors' affiliations

.....................

Correspondence to: Dr V Paradis, Service d'Anatomie Pathologique, Hôpital Beaujon, 110 bd du général Leclerc, 92118 Clichy Cedex, France; vparadis@teaser.fr

Revised version received 18 May 2005 Accepted for publication 31 May 2005 Published online first 14 September 2005

Background: Budd-Chiari syndrome (BCS) is associated with parenchymal changes leading to major architecture remodelling. In order to gain further insight into the pathogenesis of BCS, we investigated expression of a set of genes involved in the course of chronic liver diseases.

Methods: Quantitative expression of 35 selected genes involved in extracellular matrix regulation, growth factors, and angiogenesis was investigated in 13 cases of BCS and compared with 10 normal livers and 13 cirrhosis cases by real time reverse transcription-polymerase chain reaction. Differential gene expression was considered significant for genes showing at least a twofold variation, with $p<0.05$.

Results: Expression of 14 genes was significantly increased in BCS versus normal liver, with the highest increase in superior cervical ganglion 10 (SCG10) gene. BCS cases were classified according to their evolution and morphological pattern as either acute or chronic in six and seven cases, respectively. Unsupervised hierarchical clustering of acute and chronic BCS cases on the basis of similarity in gene expression pattern led to distinction between the two groups. Expression of three genes was significantly different in acute versus chronic BCS (increase in matrix metalloproteinase 7 and SCG10, decrease in thrombospondin-1 for chronic BCS). Seventeen and 10 genes, mainly involved in extracellular matrix and vascular remodelling, were significantly deregulated in acute BCS versus normal liver and cirrhosis, respectively.

Conclusion: These results show that BCS cases display a specific gene expression profile that is different from that of normal liver and cirrhosis; the molecular configuration of BCS can be readily distinguished by its evolution and morphological pattern.

B udd-Chiari syndrome (BCS) is a rare liver disease related to obstruction of the hepatic venous system. It is a clinically heterogeneous disorder, presenting as an acute, subacute, or chronic liver disease, and occurs predominantly in young adults. ${ }^{1}$ A recent pathological report of explanted livers focusing on parenchymal changes in the course of severe BCS clearly demonstrated the importance of superimposed portal flow obstruction in the progression of the disease, and the relationship between the increase in hepatic arterial perfusion and the development of large regenerative nodules. ${ }^{23}$

Liver fibrosis is the hallmark of all chronic liver diseases, irrespective of their origin. However, on histology, elementary morphological features may vary according to a specific aetiology. In addition, fibrogenesis in specific settings is clearly associated with an inflammatory reaction within the portal tract and lobule. Indeed, this inflammatory reaction is one of the key mechanisms triggering fibrogenesis, through production of various cytokines and growth factors. ${ }^{45}$ In chronic vascular diseases, including BCS, extracellular matrix accumulation in the liver follows a different stepwise progression, with fibrosis initially developing around the centrolobular veins and progressing towards extensive fibrous septa interconnecting different vascular structures, with no significant inflammation. ${ }^{67}$ These morphological data suggest that liver fibrogenesis requires additional or different mechanisms in the context of BCS. Among them, hypoxia induced cytokines involved in angiogenesis remodelling or extracellular matrix production might be relevant candidates. ${ }^{8}$ Finally, it has been shown from animal models and in vitro studies that connective tissue growth factor (CTGF), a member of the CCN (Connective tissue growth factor/fisp 12, Cyr61/cef10 and Nov) family, may be one of the main profibrogenic mediators involved in the development of fibrosis following the occurrence of vascular damage. ${ }^{9-11}$

Therefore, the mechanisms and gene pathways that trigger fibrogenesis in BCS might be different from those observed in post-inflammatory fibrosis, such as viral hepatitis, or autoimmune or biliary diseases. The objective of the present study was to gain further insight into the pathogenesis of BCS using a molecular approach to assess deregulation of some key genes potentially involved in the course of this syndrome, and to focus on their longitudinal evolution. For this purpose, we investigated expression of a set of selected genes involved in various biological processes known to be implicated in liver disorders, using real time reverse transcription-polymerase chain reaction (RT-PCR). We compared a series of BCS liver specimens at various stages with normal livers and cases of cirrhosis of non-vascular origin.

\section{MATERIALS}

\section{Liver specimens}

Liver explants were obtained from 13 patients transplanted for BCS in one institution (Hôpital Beaujon, Clichy, France). Indications for liver transplantation were severe clinical manifestations uncontrolled by medical or surgical

Abbreviations: BCS, Budd-Chiari syndrome; CTGF, connective tissue growth factor; RT-PCR, reverse transcription-polymerase chain reaction; TBP, TATA box binding protein 


\begin{tabular}{|c|c|}
\hline Gene (Alternate symbol) & Gene definition \\
\hline \multicolumn{2}{|l|}{ Extracellular matrix } \\
\hline COLIAl & Collagen type I alpha 1 \\
\hline COLAAT & Collagen type IV alpha 1 \\
\hline CTGF & Connective tissue growth factor \\
\hline MMP2 & Matrix metalloproteinase 2 \\
\hline MMP7 & Matrix metalloproteinase 7 \\
\hline THBS1 & Thrombospondin-1 \\
\hline \multicolumn{2}{|l|}{ Coagulation cascade } \\
\hline$P L G$ & Plasminogen \\
\hline PLAU & Plasminogen activator urokinase \\
\hline PLAUR & Plasminogen activator urokinase receptor \\
\hline PLAT & Plasminogen activator tissue \\
\hline F3 (TF) & Tissue factor \\
\hline F2R (TR, PARI) & Thrombin receptor \\
\hline \multicolumn{2}{|c|}{ Growth factor and receptors } \\
\hline TGFB 1 & Transforming growth factor beta 1 \\
\hline PDGFA & Platelet derived growth factor alpha chain \\
\hline PDGFB & Platelet derived growth factor beta chain \\
\hline PDGFRA & Platelet derived growth factor receptor alpha \\
\hline PDGFRB & Platelet derived growth factor receptor beta \\
\hline $1 \mathrm{~L}-6$ & Interleukin 6 \\
\hline TNF & Tumour necrosis factor \\
\hline \multicolumn{2}{|c|}{ Angiogenesis and stress hypoxia } \\
\hline VEGFA & Vascular endothelial growth factor $\mathrm{A}$ \\
\hline VEGFB & Vascular endothelial growth factor B \\
\hline VEGFC & Vascular endothelial growth factor C \\
\hline VEGFD & Vascular endothelial growth factor D \\
\hline VEGFR1 & Vascular endothelial growth factor receptor 1 \\
\hline VEGFR2 & Vascular endothelial growth factor receptor 2 \\
\hline VEGFR3 & Vascular endothelial growth factor receptor 3 \\
\hline ANGPTI & Angiopoietin 1 \\
\hline ANGPT2 & Angiopoietin 2 \\
\hline TEK (TIE2) & TEK tyrosine kinase \\
\hline HIFIA & Hypoxia inducible factor 1 alpha \\
\hline THBD & Thrombomodulin \\
\hline XLKDI (LYVEI) & Lymphatic vascular endothelial receptor 1 \\
\hline NOS3 (ENOS) & Endothelial nitric oxide synthase \\
\hline \multicolumn{2}{|l|}{ Cytoskeletal molecules } \\
\hline KRT19 & Keratin 19 \\
\hline STMN2 (SCG10) & Superior cervical ganglion 10 \\
\hline
\end{tabular}

management and/or liver failure (defined as factor $\mathrm{V}<40 \%$ ). Duration of BCS was assessed as the time from the onset of clinical manifestations of BCS to the date of transplantation. All patients were tested for thrombophilic factors, including primary myeloproliferative disorders (patent or occult), factor V Leiden, prothrombin gene G 20210 A mutation, deficiencies in protein $\mathrm{C}$, protein $\mathrm{S}$, or antithrombin, and presence of antiphospholipid antibodies.

Histological analysis was performed on liver explants using extensive samplings throughout the entire liver. Morphological elementary features systematically evaluated were: (1) ischaemic necrosis (considered significant when more than $20 \%$ of hepatocyte necrosis was present); (2) fibrosis scored on a four point scale (0, no fibrosis; 1 , venocentric fibrosis without bridging; 2 , extensive venocentric fibrosis with bridging; 3, numerous venoportal bridging; 4, "venoportal cirrhosis"); (3) nodular regenerative hyperplasia, defined as multiple regenerative nodules without a circumscribed fibrous rim $(0$, absent; 1 , focal; 2, extensive); and (4) the presence of liver macronodules (0, absent; l, present). BCS cases were separated into two groups (acute and chronic BCS) according to the presence of extensive fibrosis (fibrosis stage $\geqslant F 2$ ) in the native liver.

As controls, 13 cases of liver explants displaying cirrhosis related to chronic hepatitis $\mathrm{C}$ or $\mathrm{B}$ virus infection or chronic alcohol consumption and 10 histologically normal livers obtained from surgical specimens with benign tumours or metastasis were included.
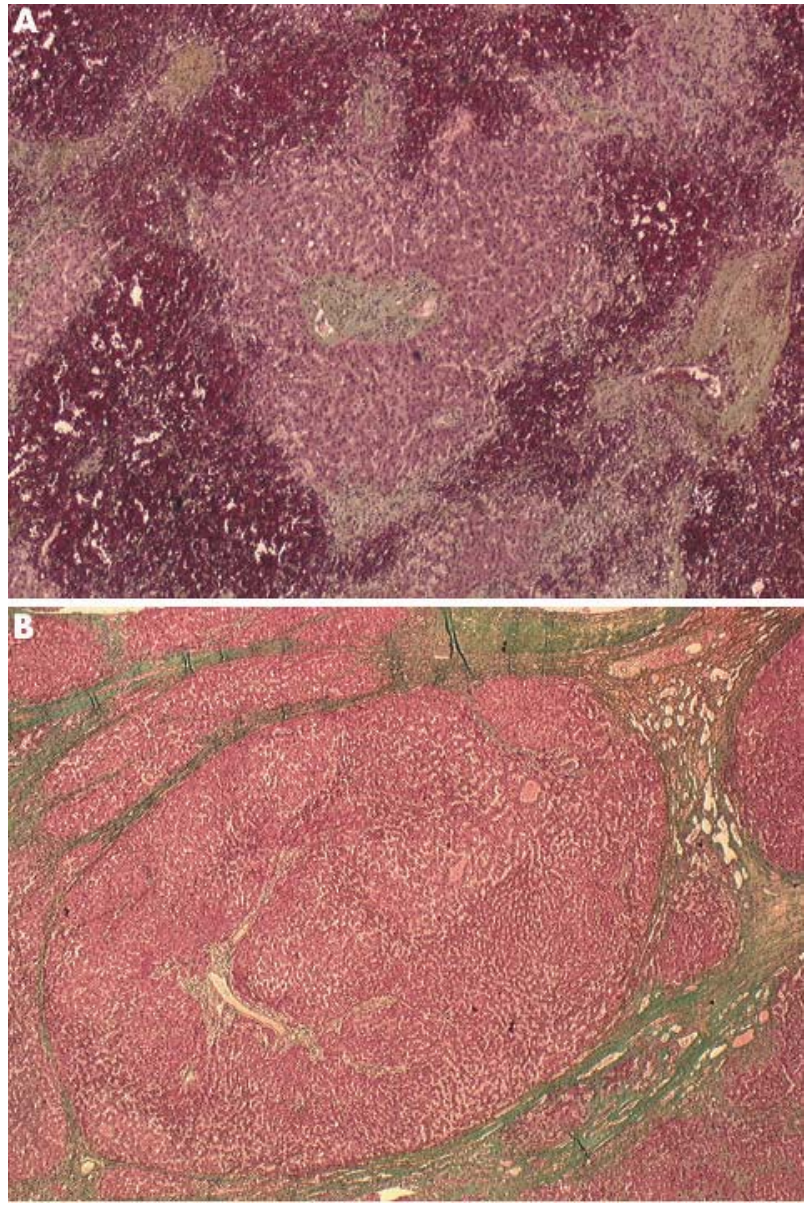

Figure 1 Morphological patterns of acute (A) and chronic (B) BuddChiari syndrome. (A) Case No 32: extensive ischaemic and congestive necrosis of centrolobular areas without fibrosis. Note centolobular thrombosis (*). (B) Case No 16: extensive fibrosis (score 4), termed "venoportal cirrhosis". Note the portal tract included in the nodule (**).

\section{Real time RT-PCR}

A total of 35 genes, selected based on their role mainly in the process of fibrogenesis and angiogenesis, were quantified. Genes are listed in table 1. Total RNA was extracted from BCS, cirrhotic, and normal livers, using the acid phenol guanidium method. All steps of the real time RT-PCR procedure have been previously described. ${ }^{12}$ We quantified transcripts of the TBP (TATA box binding protein) gene as the endogenous RNA control. Each sample was normalised on the basis of TBP content. Results, expressed as $\mathrm{N}$-fold differences in target gene expression relative to the TBP gene (termed $\mathrm{N}^{\text {target }}$ ), were determined by the following formula:

$$
\mathrm{N}^{\text {target }}=2^{\Delta \text { Ctsample }}
$$

where the $\Delta \mathrm{Ct}$ value of the sample was determined by subtracting the average $\mathrm{Ct}$ value of the target gene from the average Ct value of the TBP gene. $\mathrm{N}^{\text {target }}$ values of the samples were subsequently normalised such that the mean ratio of the normal liver would equal a value of 1 . Experiments were performed with duplicates for each data point.

\section{Statistical analysis}

For comparison of gene expression levels, we used the nonparametric Mann-Whitney and an unpaired two sample $t$ test. Genes were considered to be differentially expressed when the mean expression level was higher than a twofold difference between groups with a p value $<0.05$. 
Table 2 Clinicopathological data of the 13 patients with Budd-Chiari syndrome

\begin{tabular}{|c|c|c|c|c|c|c|c|}
\hline $\begin{array}{l}\text { Case } \\
\text { No }\end{array}$ & Sex & $\begin{array}{l}\text { Age } \\
\text { (y) }\end{array}$ & Aetiology & Evolution & $\begin{array}{l}\text { Fibrosis } \\
\text { stage }\end{array}$ & $\begin{array}{l}\text { Liver } \\
\text { macronodules }\end{array}$ & $\begin{array}{l}\text { Nodular } \\
\text { regenerative } \\
\text { hyperplasia }\end{array}$ \\
\hline 32 & $M$ & 48 & $M D$ & A & 0 & 0 & 1 \\
\hline 22 & $M$ & 32 & FC & A & 0 & 0 & 1 \\
\hline 16 & $\mathrm{~F}$ & 43 & Un & C & 4 & 1 & 1 \\
\hline 31 & $\mathrm{~F}$ & 34 & $M D$ & C & 2 & 1 & 2 \\
\hline 20 & $\mathrm{~F}$ & 34 & $M D$ & C & 3 & 1 & 1 \\
\hline 27 & $\mathrm{~F}$ & 42 & $\mathrm{MD} / \mathrm{FC}$ & $A$ & 0 & 0 & 1 \\
\hline 25 & $F$ & 22 & $M D / F C$ & A & 0 & 0 & 1 \\
\hline 24 & $M$ & 30 & $M D$ & C & 3 & 1 & 1 \\
\hline 12 & $\mathrm{~F}$ & 30 & $\mathrm{FC}$ & C & 3 & 1 & 1 \\
\hline 21 & $\mathrm{~F}$ & 33 & $M D$ & C & 3 & 1 & 1 \\
\hline 14 & $\mathrm{~F}$ & 21 & APL & C & 2 & 1 & 2 \\
\hline 9 & $F$ & 38 & MD/PS & A & 1 & 0 & 2 \\
\hline 4 & M & 21 & FC & A & 0 & 0 & 3 \\
\hline
\end{tabular}

BRB-ArrayTools Ver.3.1.0 (http://linus.nci.nih.gov/BRBArrayTools.html) was used to compare gene expression levels, class prediction, hierarchical clustering, and multidimensional scaling of samples.

\section{RESULTS}

\section{Clinical and pathological data}

Patients with BCS included nine women and four men, with a mean age of 33 years (range 2l-48). The mean interval between the diagnosis of BCS and liver transplantation was 42 months (range 0.5-120 months). The pretransplant course was six months or less in five patients. Primary myeloproliferative disorders were identified in eight patients (three also displayed a thrombophilic condition), factor $\mathrm{V}$ Leiden in two, a prothrombin gene mutation in one, isolated antiphospholipid antibodies in one, and no detectable prothrombotic disorder was found in one patient. Extensive samplings of the native livers showed both significant fibrosis (stage $\geqslant 2$ ) and the presence of liver macronodules in seven cases.

Nodular regenerative hyperplasia features were observed in all native livers, restricted to some areas in nine cases and extensively distributed in four cases. According to morphological features (presence of extensive fibrosis), cases were classified as either acute BCS $(n=6)$ or chronic BCS $(n=7)$. Typical cases of acute and chronic BCS are illustrated in fig 1. Mean duration of symptoms in the group of acute and

Table 3 Genes with significant differential levels of expression (twofold difference and $p<0.05$ ) in BuddChiari syndrome versus normal liver

\begin{tabular}{|c|c|c|}
\hline Gene & $\begin{array}{l}\mathrm{N} \text { fold versus normal } \\
\text { liver }\end{array}$ & p Value \\
\hline SCG10 & $13.3(4.3)$ & 0.02 \\
\hline CTGF & $9.3(1.9)$ & 0.001 \\
\hline COLIAI & $9.1(2.4)$ & 0.001 \\
\hline F3 & $7.4(1.9)$ & 0.002 \\
\hline ANGPT1 & $6.7(1.0)$ & 0.0001 \\
\hline PDGFRA & $6.2(1.6)$ & 0.001 \\
\hline COL4A 1 & $5.7(1.1)$ & 0.0008 \\
\hline MMP2 & $5.4(1.0)$ & 0.0001 \\
\hline PLAT & $5.0(0.9)$ & 0.001 \\
\hline PLAU & $4.3(1.0)$ & 0.009 \\
\hline PDGFA & $4.1(0.6)$ & 0.0006 \\
\hline PDGFRB & $4.0(0.8)$ & 0.003 \\
\hline VEGFC & $3.1(0.7)$ & 0.01 \\
\hline PDGFB & $2.9(0.6)$ & 0.009 \\
\hline
\end{tabular}

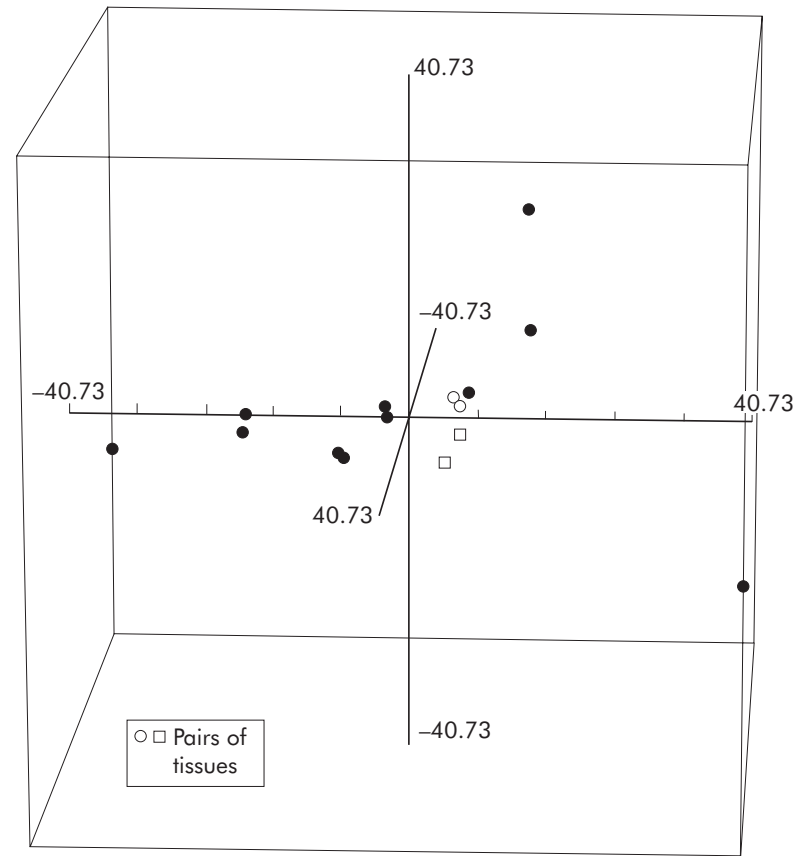

Figure 2 Three dimensional scaling plot of the 15 Budd-Chiari syndrome samples obtained from 13 patients using the 35 genes. Pairs of tissue samples are shown.

chronic BCS was 2.3 and 61 months, respectively. Clinical and pathological data are reported in table 2 .

Histologically, normal livers were obtained from surgical samples at a distance from the liver metastasis $(n=4)$ or benign tumours (liver cell adenoma, $\mathrm{n}=\mathrm{l}$; focal nodular hyperplasia, $\mathrm{n}=1$; biliary cyst, $\mathrm{n}=1$; and haemangioma, $\mathrm{n}=3$ ) in 10 patients (eight women and two men; mean age 53 years). Cirrhotic liver samples were obtained from 13 patients ( 13 men; mean age 60 years) with chronic hepatitis C infection $(\mathrm{n}=8)$, chronic hepatitis B infection $(\mathrm{n}=3)$, and chronic alcohol consumption $(\mathrm{n}=2)$.

\section{Differential gene expression in BCS versus normal} liver

Relative RNA expression level of the 35 selected genes was quantified by real time RT-PCR and compared between the groups of BCS (including both acute and chronic cases) and normal liver. As significant morphological heterogeneity was observed throughout the entire liver, serial liver samples 


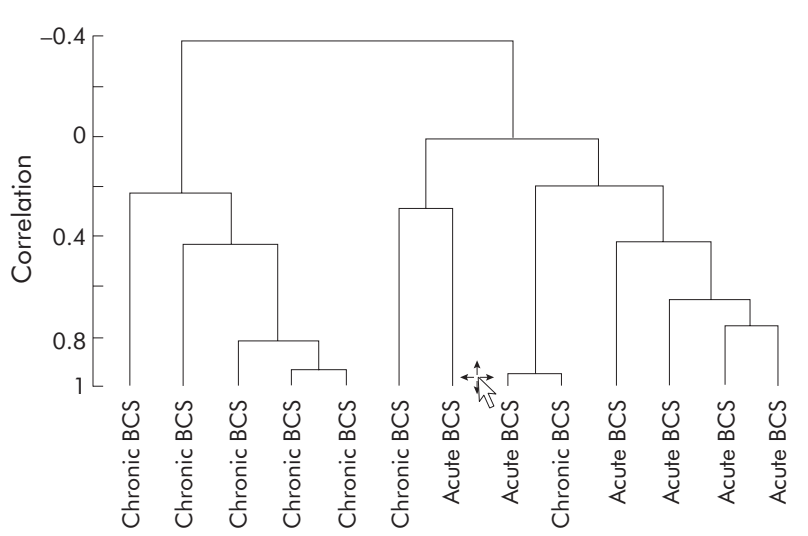

Figure 3 Unsupervised hierarchical clustering of acute and chronic Budd-Chiari syndrome (BCS) according to 35 gene expression profiles. On the basis of similarity in gene expression profiles, two main branches, one with the acute cases (acute BCS) and the other with chronic cases (chronic BCS), were observed.

obtained from two BCS cases were included. Thus a total of 15 BCS samples from 13 patients were used for the study. Results were normalised to the level of expression of each gene in the group of normal livers. Significant differential expression in BCS, as high as twofold the value of normal liver, was observed for 14 of the 35 genes tested, with upregulation for all of them in the BCS group. The highest increase in gene expression level was observed for a molecule belonging to the OP18/stathmin family (SCG10). Detailed data are given in table 3 .

In order to check for possible heterogeneity within the liver, gene expression was analysed in two separate samples performed either in the left or right lobe of two native livers (case Nos 9 and 14). A three dimensional scaling plot was performed using the 35 genes and BRB array tools. This representation assesses the position of each case in three dimensional Euclidean space with the distance between each data point reflecting their approximate degree of correlation. Each pair of tissue samples provided from the same liver clustered together, as shown in fig 2, suggesting that heterogeneity is not a relevant limit in the present study.

\section{Gene expression pattern of BCS according to evolution of the disease}

BCS cases included in the study were separated into two groups (acute and chronic BCS) according to their morphological pattern. An unsupervised hierarchical clustering of cases with acute and chronic BCS on the basis of similarity in the gene expression pattern yielded two major clusters, one representing acute BCS and one representing chronic BCS, with only two exceptions. A representative dendrogram is shown in fig 3. Thus BCS can be readily distinguished not only according to its clinical evolution but also to its molecular configuration. Therefore, expression levels of the 35 selected genes were compared between the acute and chronic BCS groups. This analysis showed differential gene expression for three genes (SCG10, MMP7, and THBS1), with two upregulated in the group of chronic versus acute BCS. One of the upregulated genes, MMP7, is mainly involved in remodelling of the extracellular matrix, and the highest mRNA increase was observed for SCG10. In contrast, THBSI was significantly downregulated during the course of evolution of BCS. Data are given in table 4 .

\section{Differential gene expression in acute and chronic BCS versus normal liver}

In order to focus on genes involved early in the course of BCS, we compared gene expression levels in the acute BCS group
Table 4 Genes differentially expressed in Budd-Chiari syndrome (BCS) as a function of evolution of the disease (acute versus chronic BCS)

\begin{tabular}{llcl}
\hline Gene & Acute BCS & Chronic BCS & p Value \\
\hline SCG10 & $2.9(1.3)$ & $25.1(7.0)$ & 0.002 \\
MMP7 & $1.2(0.3)$ & $5.1(0.9)$ & 0.002 \\
THBS1 & $3.1(0.7)$ & $1.3(0.3)$ & 0.03 \\
\hline \multicolumn{4}{l}{ Mean values in acute and chronic BCS versus normal liver. }
\end{tabular}

Table 5 Differential gene expression in Budd-Chiari syndrome (acute or chronic BCS) versus normal liver

\begin{tabular}{|c|c|c|c|c|}
\hline \multirow[b]{2}{*}{ Gene } & \multicolumn{2}{|l|}{ Acute BCS } & \multicolumn{2}{|c|}{ Chronic BCS } \\
\hline & Mean (SD) & p Value & Mean (SD) & p Value \\
\hline COLIAI & $7.5(1.1)$ & $<0.0001$ & $10.9(5.2)$ & 0.04 \\
\hline ANGPTI & $5.8(0.8)$ & $<0.0001$ & $7.9(2.0)$ & 0.0009 \\
\hline COL4AI & $4.8(0.7)$ & $<0.0001$ & $6.7(2.4)$ & 0.01 \\
\hline MMP2 & $4.1(0.8)$ & 0.001 & $6.9(2.0)$ & 0.003 \\
\hline PDGFRB & $3.3(0.8)$ & 0.007 & $4.8(1.5)$ & 0.009 \\
\hline PLAT & 4.9 (1.4) & 0.008 & $5.2(1.3)$ & 0.001 \\
\hline CTGF & $11.4(2.6)$ & 0.0004 & $6.9(2.8)$ & 0.02 \\
\hline PDGFA & $3.3(0.8)$ & 0.007 & $4.9(0.9)$ & 0.0003 \\
\hline PDGFRA & $4.1(0.5)$ & $<0.0001$ & $8.5(3.4)$ & 0.02 \\
\hline PLAU & $4.8(1.6)$ & 0.02 & $3.8(1.2)$ & 0.02 \\
\hline F3 & $6.8(1.1)$ & $<0.0001$ & $8.0(4.0)$ & 0.05 \\
\hline PDGFB & $2.5(0.4)$ & 0.003 & $3.3(1.3)$ & 0.05 \\
\hline IL6 & $4.3(1.5)$ & 0.03 & $2.8(2.4)$ & NS \\
\hline VEGFC & $3.1(0.9)$ & 0.02 & $1.0(0.3)$ & NS \\
\hline THBSI & $3.1(0.7)$ & 0.005 & $1.2(0.4)$ & NS \\
\hline ANGPT2 & $2.6(0.5)$ & 0.007 & $4.6(3)$ & NS \\
\hline$T H B D$ & $2.2(0.5)$ & 0.03 & $1.3(0.3)$ & NS \\
\hline SCG10 & $2.9(1.3)$ & NS & $25.1(7.0)$ & 0.0009 \\
\hline MMP7 & $1.2(0.3)$ & NS & $5.1(0.9)$ & 0.001 \\
\hline
\end{tabular}

versus normal liver. Among 35 selected genes, significant differential expression was observed for 17 genes, all of which were upregulated in the acute BCS group. Several genes involved in extracellular matrix (COL1A1, COL4Al, $C T G F$, and $M M P 2$ ) or vascular (ANGPT1 and 2) remodelling, and tissular factor $(F 3)$ were among the most discriminating $(p<0.001)$, enabling accurate classification of $94 \%$ of cases using a leave-one-out cross validation procedure and several different models (nearest neighbour, compound covariate predictor, nearest centroid or support vector Machn). Interestingly, genes involved in the coagulation cascade were also significantly upregulated (PLAU and PLAT). Quantitative data are given in table 5 .

In order to focus on genes whose deregulation was maintained at the stage of chronic BCS, gene expression levels were compared between chronic BCS and normal liver. Fourteen genes were significantly upregulated in chronic BCS versus normal liver (table 5 ).

In total, this approach led to distinction of specific profiles of gene expression according to the evolution of the disease. Group 1, defined by genes upregulated in acute BCS and which returned to normal levels at the chronic stage, included PDGFRA, PDGFA, ANGPT2, VEGFC, IL-6, and THBD. Group 2, defined by genes upregulated in acute BCS and remaining at a stable high level in the chronic stage, included $A N G P T 1$, COLIA1, F3, COL4A1, CTGF, PDGFB, PDGFRB, PLAU, and $M M P 2$. Group 3, defined by genes which increased only at the chronic stage, included MMP7 and SCG10.

\section{Differential gene expression in chronic BCS versus cirrhotic liver}

In chronic BSC, venous outflow obstruction leads to the development of perivenular and perisinusoidal fibrosis in 
Table 6 Differential gene expression in chronic BuddChiari syndrome (BCS) versus cirrhosis

\begin{tabular}{llll}
\hline Gene & Chronic BCS & Cirrhosis & p Value \\
\hline PDGFRA & $8.5(3.4)$ & $2.4(0.4)$ & 0.02 \\
ANGPT1 & $7.9(2.0)$ & $1.6(0.2)$ & 0.0004 \\
CTGF & $6.9(2.8)$ & $2.0(0.4)$ & 0.03 \\
MMP2 & $6.9(2.0)$ & $3.2(2.1)$ & 0.03 \\
COL4A1 & $6.7(2.4)$ & $1.8(0.3)$ & 0.01 \\
PLAT & $5.1(1.3)$ & $1.7(0.3)$ & 0.003 \\
PDGFA & $4.9(1.0)$ & $2.3(0.4)$ & 0.008 \\
PDGFB & $3.3(1.3)$ & $1.1(0.2)$ & 0.02 \\
VEGFR3 & $2.0(0.4)$ & $0.7(0.1)$ & 0.0007 \\
KRT19 & $1.4(0.4)$ & $4.5(0.9)$ & 0.02
\end{tabular}

Mean values in chronic Budd-Chiari syndrome and cirrhosis versus normal liver.

centrolobular areas, which further progresses to extensive fibrosis and cirrhosis. In order to assess whether specific regulation pathways may be involved in vascular fibrosis and cirrhosis, differential gene expression was investigated in the chronic BCS group versus cirrhotic samples of other origins. This analysis showed that 10 genes were significantly deregulated, with nine upregulated in the chronic BCS group versus cirrhotic samples. Most of them encoded extracellular matrix genes or associated molecules (COL4AI, CTGF, and $M M P 2)$, angiogenic molecules (ANGPT1 and VEGFR3), profibrogenic cytokines (PDGFA, PDGFB, and PDGFRA), and PLAT. The downregulated gene in chronic BCS was related to an epithelial marker of biliary cells (KRT19). Data are reported in table 6. Interestingly, the three multidimensional scaling plot showed complete separation between the chronic BCS group and that of cirrhosis (fig 4).

\section{DISCUSSION}

To our knowledge, this is the first study of the pathogenesis of liver parenchymal changes related to BCS using a gene expression approach. Although the molecular investigation was limited to a restricted set of genes, this approach provided further insight into the biological and cellular mechanisms involved in the development of liver injury associated with BCS. Using a real time RT-PCR approach, we

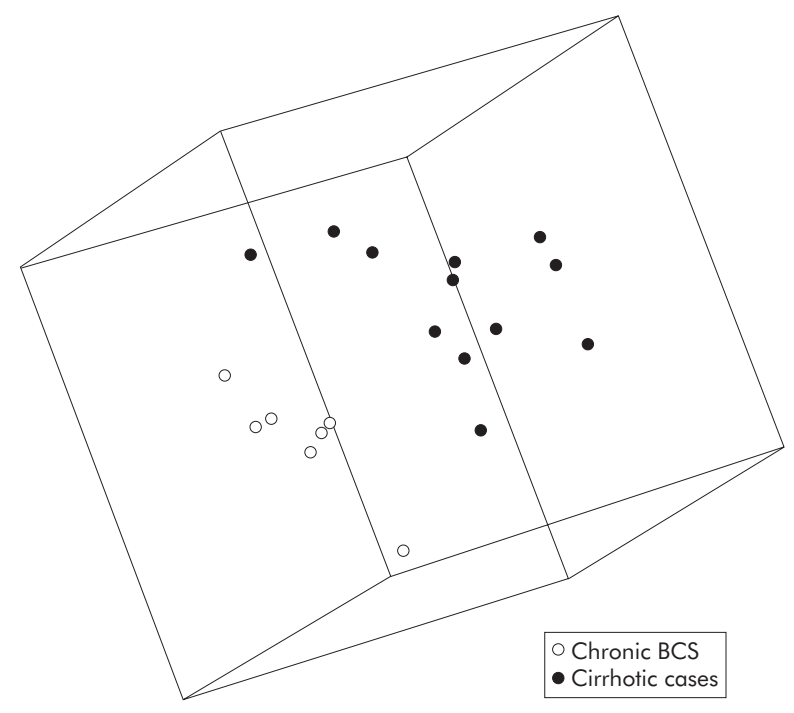

Figure 4 Three dimensional scaling plot of chronic Budd-Chiari syndrome (BCS) and cirrhosis. This representation assesses the position of each case in three dimensional space. The distance between each data point reflects their approximate degree of correlation. Chronic BCS and cirrhotic cases are shown. quantified a set of genes in a series of native livers displaying pathological features of BCS representative of the different stages of the disease. Our choice of genes was based on molecular knowledge of the main metabolic pathways involved in fibrogenesis, focusing on genes that are mainly regulated at the transcriptional level. Thus we identified several genes displaying significant differential expression in BCS compared with normal tissue or cirrhosis, and also within the group of BCS according to evolution.

Firstly, comparison between BCS as a whole and normal liver showed that a set of genes was upregulated in BCS. As expected, most are involved in the process of extracellular matrix remodelling (COL1A1, COL4A1, CTGF, and MMP2), vasculogenic molecules (ANGPT1,VEGFC), or growth factors and their receptors (PDGFA, PDGFB, PDGFRA, and PDGFRB). Interestingly, the highest mRNA upregulation in BCS was observed for SCG10, a gene known to be restricted to neural cells. Indeed, SCG10 belongs to the OP18/stathmin family and was initially identified as a marker of neuronal differentiation. ${ }^{13}{ }^{14}$ Like other members of the stathmin family, SCG10 functions as a tubulin sequester, interfering with microtubule dynamics and thus playing a role in cell motility. ${ }^{15}{ }^{16}$ Upregulation of SCG10 in the BCS group, and especially in the chronic BCS group displaying significant fibrosis, is in accordance with recent experimental and human data showing gradual upregulation of SCG10 mRNA in the development of liver fibrosis. ${ }^{17}$

In order to gain further insight into the pathogenesis of early stages of BCS, we compared gene expression profiles of acute BCS with that of normal liver. Results showed that, in addition to the expected extracellular matrix and vascular genes, tissue factor (F3) was significantly upregulated in acute BCS. F3, a major regulator of coagulation through binding of factor VII, is mainly expressed, under physiological conditions, by cells not in contact with blood, including vascular smooth muscle, mesenchymal cells, and epithelial cells. Nevertheless, induction of F3 expression by endothelial cells has been shown to contribute to intravascular thrombosis. ${ }^{18}$ Based on our results, it can be hypothesised that during BCS, upregulation of F3 mRNA expression in the liver may participate in or be associated with subsequent venous outflow obstruction. In addition, F3 has recently been recognised as an inducer of angiogenesis through upregulation of VEGF, especially in the context of tumour invasion and metastasis. ${ }^{19} 20$ Interestingly, in this study, we also observed a significant increase in VEGF mRNA in acute BCS versus normal liver.

Liver fibrosis is a hallmark of chronic liver disease, although some morphological features are suggestive of a specific aetiology. In this regard, we sought to compare the gene expression pattern of chronic BCS, characterised by extensive fibrosis in the absence of significant inflammation, with liver cirrhosis of other origin. This analysis demonstrated that both extracellular matrix and vasculogenesis encoding genes (COLIAl, COL4AI, and ANGPTI) were expressed at higher levels in the context of chronic BCS than in alcoholic or viral induced cirrhosis. These data may reflect extensive remodelling of the mesenchymal compartment that occurs in BCS. Interestingly, the highest increase in mRNA expression observed in chronic BCS versus cirrhosis was that of CTGF. CTGF is a potent cystein rich heparin binding growth factor, initially isolated from human umbilical vein endothelial cells, that is also highly expressed in mesenchymal cells, including fibroblasts and hepatic stellate cells..$^{21-23}$ Previous studies have provided evidence that CTGF may play a significant role in promoting extracellular matrix production after tissue injury, including liver fibrosis, by stimulating type 1 collagen and fibronectin mRNA expression. ${ }^{24}{ }^{25}$ Interestingly, Chambers et al showed that CTGF expression 
may be induced by molecules involved in the coagulation cascade, such as thrombin. ${ }^{26}$ Indeed, the BCS syndrome is related to genetic or acquired prothrombotic conditions which results in random obstructions within the venous hepatic system. Therefore, it is conceivable that the formation of multiple venous thrombosis, observed in the course of this disease, may directly participate in the further development of liver fibrogenesis, partly through upregulation of CTGF expression. Such a mechanism would also explain recent findings that, in the context of chronic viral hepatitis, there is a significant association between advanced fibrosis and underlying thrombophilic states. ${ }^{27}{ }^{28}$ The molecular relevance of these clinical data is provided by in vitro studies which showed that thrombin receptors are upregulated in hepatic stellate cells during liver damage. ${ }^{29}{ }^{30}$ Finally, an additional factor potentially involved in CTGF upregulation observed in chronic BCS may be the mechanical stress that is induced in the sinusoidal and perisinusoidal spaces of the liver secondary to venular obstructions. Indeed, it has been clearly shown that CTGF displays the most striking upregulation, among 588 different cDNAs using gene array technology, induced by mechanical stress in human lung fibroblasts. ${ }^{31}$ Finally, as no adjustments for mass significance were made, conclusions must be drawn with caution. However, both internal consistency, level of gene expression variation, and biological plausibility strengthen the argument that the effect of mass is small.

In conclusion, this study shows that fibrogenic and angiogenic factors are differentially expressed in BCS livers compared with cirrhosis of other origin. Furthermore, this study points to differential expression of these factors according to stage of disease.

\section{ACKNOWLEDGEMENT}

The study was supported by grants from SNFGE (Société Nationale Française de Gastroentérologie).

\section{Authors' affiliations \\ V Paradis, P Bedossa, Service d'Anatomie Pathologique, Hôpital} Beaujon, Clichy, France, and CNRS UMR 8149, Faculté de Pharmacie, Paris, France

I Bièche, I Laurendeau, M Vidaud, UPRES EA 3618, Faculté de

Pharmacie, Paris, France

D Dargère, CNRS UMR 8149, Faculté de Pharmacie, Paris V, France

D Cazals-Hatem, Service d'Anatomie Pathologique, Hôpital Beaujon, Clichy, France

V Saada, A Bezeaud, Service d'Hématologie, Hôpital Beaujon, Clichy, France

J Belghiti, Service de Chirurgie Hépatique, Hôpital Beaujon, Clichy, France

D-C Valla, Service d'Hépatologie, Hôpital Beaujon, Clichy, France

Conflict of interest: None declared.

\section{REFERENCES}

1 Valla D, Benhamou J. Obstruction of the hepatic venous system. In: Bircher J, Benhamou J, Mclntyre N, eds. Oxford textbook of clinical pathology, 2nd edn. Oxford: Oxford Medical Publication, 1999:1469-78.

2 Cazals-Hatem D, Vilgrain V, Genin P, et al. Arterial and portal circulation in Budd-Chiari syndrome: A study in 17 explanted livers. Hepatology 2003:37:510-19.

3 Ibarrola C, Castellano VM, Colina F. Focal hyperplastic hepatocellular nodules in hepatic venous outflow obstruction: a clinicopathological study of four patients and 24 nodules. Histopathology 2004;44:172-9.
4 Friedman SL. Liver fibrosis - from bench to bedside. J Hepatol 2003;38(suppl):53.

5 Fontaine $H$, Nalpas B, Poulet B, et al. Hepatitis activity index is a key factor in determining the natural history of chronic hepatitis $C$. Hum Pathol 2001;32:904-9.

6 Tanaka M, Wanless IR. Pathology of the liver in Budd-Chiari syndrome: portal vein thrombosis and the histogenesis of veno-centric cirrhosis, veno-portal cirrhosis, and large regenerative nodules. Hepatology 1998;27:488-96.

7 Okuda K, Kage M, Shrestha SM. Proposal of a new nomenclature for BuddChiari syndrome: hepatic vein thrombosis versus thrombosis of the inferior vena cava at its hepatic portion. Hepatology 1998;28:1191-8.

8 Corpechot C, Barbu V, Wendum D, et al. Hypoxia-induced VEGF and collagen 1 expressions associated with angiogenesis and fibrogenesis in experimental cirrhosis. Hepatology 2002;35:1010-21.

9 Riser BL, Denichillo M, Cortes P, et al. Regulation of connective tissue growth factor activity in cultured rat mesangial cells and its expression in experimental diabetic glomerulosclerosis. J Am Soc Nephrol 2000;11:25-38.

10 Finckenberg $\mathbf{P}$, Lassila $M$, Inkinen K, et al. Cyclosporine induces myocardial connective tissue growth factor in spontaneously hypertensive rats on highsodium diet. Transplantation $2001 ; 71: 951-8$.

11 Ruperez M, Lorenzo O, Blanco-Colio LM, et al. Connective tissue growth factor is a meditor of angiotensin II-induced fibrosis. Circulation 2003; 108: 1499-505.

12 Paradis V, Bièche $Y$, Dargère $D$, et al. A quantitative gene expression study suggests a role for angiopoietins in focal nodular hyperplasia. Gastroenterology 2003;124:651-9.

13 Anderson DJ, Axel R. Molecular probes for the development and plasticity of neural crest derivatives. Cell 1985;42:649-62.

14 Ozon S, Byk T, Sobel A. SCLIP: a novel SCG10-like protein of the stathmin family expressed in the nervous system. J Neurochem 1998;70:2386-96.

15 Charbaut E, Curmi PA, Ozon S, et al. Stathmin family proteins display specific molecular and tubulin binding properties. J Biol Chem $2001 ; 276: 16146-54$.

16 Curmi PA, Andersen SSL, Lachkar S, et al. The stathmin tubulin interaction in vitro. J Biol Chem 1997;272:25029-36.

17 Bièche I, Asselah T, Laurendeau I, et al. Molecular profiling of early-stage liver fibrosis in patients with chronic hepatitis $C$ virus infection. Virology 2005;332:130-144.

18 Bevilacqua MP, Gimbrone MA. Inducible endothelial functions in inflammation and coagulation. Semin Thromb Hemost 1987; 13:425-33.

19 Abe K, Shoii M, Chen J, et al. Regulation of vascular endothelial growth factor production and angiogenesis by the cytoplasmic tail of tissue factor. Proc Natl Acad Sci U S A 1999;96:8663-8.

20 Shoji M, Hancock WW, Abe K, et al. Activation of coagulation and angiogenesis in cancer: immunohistochemical localization in situ of clotting proteins and vascular growth factor in human cancer. Am J Pathol 1998;152:399-411.

21 Bradham DM, Igarashi A, Potter RL, et al. Connective tissue growth factor: a cysteine-rich mitogen secreted by human vascular endothelial cells is related to the SRC-induced immediate early gene product CEF-10. J Cell Biol 1991; 114:1285-94.

22 Igarashi A, Nashiro K, Kikuchi K, et al. Connective tissue growth factor gene expression in tissue sections from localized scleroderma, keloid, and other fibrotic skin disorders. J Invest Dermatol 1996;106:729-33.

23 Paradis V, Dargère $D$, Vidaud $M$, et al. Expression of connective tissue growth factor in experimental and human liver fibrosis. Hepatology 1999;30:968-76.

24 Frazier K, Williams S, Kothapalli D, et al. Stimulation of fibroblast cell growth, matrix production, and granulation tissue formation by connective tissue growth factor. J Invest Dermatol 1996;107:404-11.

25 Paradis V, Perlemutter G, Bonvoust F, et al. High glucose and hyperinsulinemia stimulate connective tissue growth factor expression: a potential mechanism involved in progression to fibrosis in non-alcoholic steatohepatitis. Hepatology $2001 ; 34: 738-44$.

26 Chambers RC, Leoni P, Blanc-Brude OP, et al. Thrombin is a potent inducer of connective tisue growth factor production via proteolytic activation of protease-activated receptor-1. J Biol Chem 2000;275:35584-91.

27 Papatheodoridis GV, Papakonstantinou E, Andrioti E, et al. Thrombotic risk factors and extent of liver fibrosis in chronic viral hepatitis. Gut 2003;52:404-9.

28 Wright M, Goldin R, Hellier S, et al. Factor V Leiden polymmorphism and the rate of fibrosis development in chronic hepatitis $C$ virus infection. Gut 2003;52:1206-10.

29 Marra F, DeFranco R, Grappone C, et al. Expression of the thrombin receptor in human liver: Up-regulation during acute and chronic injury. Hepatology 1998;27:462-71.

30 Duplantier JG, Dubuisson L, Senant L, et al. A role for thrombin in liver fibrosis. Gut 2004;53:1682-7.

31 Schild C, Trueb B. Mechanical stress is required for high level expression of connective tissue growth factor. Exp Cell Res 2002;274:83-91. 